\title{
A human amygdala site that inhibits respiration and elicits apnea in pediatric epilepsy
}

\author{
Ariane E. Rhone, ${ }^{1,2}$ Christopher K. Kovach, ${ }^{1,2}$ Gail I.S. Harmata, ,2,3,4,5,6 Alyssa W. Sullivan, ${ }^{7}$ \\ Daniel Tranel, ${ }^{2,7,8}$ Michael A. Ciliberto, ${ }^{9}$ Matthew A. Howard, ${ }^{1,2,3}$ George B. Richerson, ${ }^{2,3,4,8,10}$ \\ Mitchell Steinschneider, ${ }^{11}$ John A. Wemmie, ${ }^{1,2,3,4,6,8,10,12}$ and Brian J. Dlouhy ${ }^{1,2,3}$ \\ 'Department of Neurosurgery, ${ }^{2}$ lowa Neuroscience Institute, ${ }^{3}$ Pappajohn Biomedical Institute, ${ }^{4}$ Interdisciplinary Graduate \\ Program in Neuroscience, ${ }^{5}$ Pharmacological Sciences Training Program, ${ }^{6}$ Department of Psychiatry, ${ }^{7}$ Department of \\ Psychological and Brain Sciences, ${ }^{8}$ Department of Neurology, ${ }^{9}$ Department of Pediatrics, and ${ }^{10}$ Department of Molecular \\ Physiology and Biophysics, Carver College of Medicine, University of lowa, lowa City, lowa, USA. "Department of \\ Neurology, Albert Einstein College of Medicine, New York, New York, USA. ${ }^{12}$ Department of Veterans Affairs Medical \\ Center, lowa City, lowa, USA.
}

BACKGROUND. Seizure-induced inhibition of respiration plays a critical role in sudden unexpected death in epilepsy (SUDEP). However, the mechanisms underlying seizure-induced central apnea in pediatric epilepsy are unknown.

METHODS. We studied 8 pediatric patients with intractable epilepsy undergoing intracranial electroencephalography. We recorded respiration during seizures and during electrical stimulation mapping of $\mathbf{1 7 4}$ forebrain sites. A machine-learning algorithm was used to delineate brain regions that inhibit respiration.

RESULTS. In 2 patients, apnea coincided with seizure spread to the amygdala. Supporting a role for the amygdala in breathing inhibition in children, electrically stimulating the amygdala produced apnea in all 8 subjects (3-17 years old). These effects did not depend on epilepsy type and were relatively specific to the amygdala, as no other site affected breathing. Remarkably, patients were unaware that they had stopped breathing, and none reported dyspnea or arousal, findings critical for SUDEP. Finally, a machine-learning algorithm based on $\mathbf{4 5}$ stimulation sites and 210 stimulation trials identified a focal subregion in the human amygdala that consistently produced apnea. This site, which we refer to as the amygdala inhibition of respiration (AIR) site includes the medial subregion of the basal nuclei, cortical and medial nuclei, amygdala transition areas, and intercalated neurons.

CONCLUSIONS. A focal site in the amygdala inhibits respiration and induces apnea (AIR site) when electrically stimulated and during seizures in children with epilepsy. This site may prove valuable for determining those at greatest risk for SUDEP and as a therapeutic target.

FUNDING. National Institute of Neurological Disorders and Stroke - Congress of Neurological Surgeons, National Institute of General Medical Sciences, Roy J. Carver Charitable Trust.

Conflict of interest: The authors have declared that no conflict of interest exists.

Copyright: (ㄷ) 2020, American Society for Clinical Investigation.

Submitted: November 13, 2019

Accepted: February 26, 2020

Published: March 12, 2020.

Reference information: JCI Insight. 2020;5(6):e134852.

https://doi.org/10.1172/jici. insight.134852.

\section{Introduction}

Sudden unexpected death in epilepsy (SUDEP) is a major health problem, recognized as the most common cause of death in patients with medically intractable epilepsy (1). Because of its increased incidence in early life compared with other neurological conditions, SUDEP ranks second only to stroke among al neurological disease in years of potential life lost (2). Recent evidence indicates SUDEP is more frequent in pediatric cases than previously thought (3). Moreover, SUDEP accounts for more than one-third of all causes of mortality in pediatric patients with epilepsy (4). Major risk factors include generalized tonic-clonic seizures (GTCS), medication intractability, and seizure frequency (5), although SUDEP can also occur in children with more typical epilepsy syndromes, such as juvenile myoclonic epilepsy, as well as children 
who have never had a GTCS (6-8). To prevent such devastating outcomes, it will be critical to identify the mechanisms underlying SUDEP in children as well as adults and in various types of epilepsy.

Although the mechanisms underlying SUDEP remain unclear, multiple lines of evidence suggest inhibition of breathing plays a critical role (9-11). Supporting this possibility, central apnea is frequently observed during seizures (12-14). Moreover, in a study of SUDEP cases that occurred in epilepsy-monitoring units, seizures resulted in terminal apnea that preceded cardiac asystole, with the resulting oxygen desaturation likely worsened by a lack of arousal and being prone, face down in bed, and without timely intervention (9). And, although sites within the brainstem are essential for breathing, a number of forebrain sites commonly affected by epilepsy have also been implicated in breathing control, including the amygdala, hippocampus, and cingulate cortex (15-17). In humans, forebrain control of breathing is thought to be important for speaking, singing, voluntary breath holding, arousal, and emotional control and expression such as anxiety, fear, panic, laughing, and crying (18-22). Forebrain sites likely influence brainstem respiratory networks through neural projections, which might provide pathways for seizure-induced apnea. Consistent with these possibilities, recent studies indicate that loss of breathing in adult patients with epilepsy coincides with seizure spread to the amygdala $(16,23)$. In adults, electrical stimulation of the amygdala reproduced this apnea $(16,17)$. Thus far, no studies have examined forebrain control of breathing in children or seizure-induced apnea in pediatric epilepsy.

Because the amygdala undergoes functional and structural connectivity changes from birth through adolescence (24-26), questions arise about the importance of the amygdala in seizure-induced apnea and SUDEP in children. First, does the amygdala play a role in seizure-induced loss of breathing in children as in adults? Second, does the amygdala inhibit breathing? Third, are these effects specific to epilepsy type? Fourth, might other forebrain sites have a similar function? And finally, do specific regions of the amygdala mediate these effects? The latter being an important possibility, as the amygdala is composed of multiple nuclei with distinct functional roles $(27,28)$. Previous studies did not delineate nucleus-specific effects on breathing, in part because they used electrodes with widely spaced contacts not suited for precise mapping $(16,17,29)$. Thus, it is unknown if stimulation of every site in the amygdala can inhibit breathing or if it is restricted to a specific site or nuclei. To answer these questions, we recorded from and functionally mapped forebrain sites in 8 pediatric patients with intractable epilepsy undergoing intracranial electroencephalography (iEEG; see Table 1 and Figure 1 for patient characteristics and electrode locations, respectively).

\section{Results}

Apnea occurs in patients with pediatric epilepsy when seizures spread to the amygdala. Six of the eight patients with pediatric epilepsy participated in 2-3 weeks of continuous respiratory monitoring while undergoing seizure mapping with intracranial electrodes. During this monitoring period, all 6 patients had interpretable respiratory signals during seizures (Table 2). Of these 6 patients, P407 and P466 had apnea during 12 of 20 captured seizures. During these 12 apneic seizures, apnea coincided with seizure spread to the amygdala. In P407, cessation of breathing occurred on average within 0.4 seconds of seizure propagation to the amygdala and prior to spread of epileptic activity to the hippocampus or other cortical sites (Table 2 and Figure 2). Similar findings were observed in P466, as cessation of breathing occurred on average within 0.7 seconds of seizure propagation to the amygdala (Table 2). In P407 and P466, in all seizures in which apnea occurred, seizure activity was observed in the amygdala. Conversely, in all patients and all seizures in which apnea did not occur, seizure activity was not observed in the amygdala.

Amygdala stimulation induced apnea in all pediatric subjects. The iEEG seizure data suggested that seizure activity within the amygdala of patients with pediatric epilepsy was associated with apnea. Although these findings indicated a relationship between the amygdala and apnea, they did not prove causation. To test whether the amygdala may be responsible for seizure-induced apnea, we electrically stimulated amygdala electrode contact sites in all 8 pediatric subjects using a bipolar biphasic stimulus (Figure 3A). Because 3 subjects had bilateral amygdala electrodes and 5 subjects had 1 amygdala electrode, in total, sites in 11 amygdalae were stimulated. In each subject, stimulation of the amygdala elicited apnea, albeit to different degrees. For example, in P381, electrical stimulation between the most medial contacts in the amygdala (contacts 1-2) (Figure 3, B and C) induced apnea that persisted throughout the duration of stimulation, beginning shortly after stimulation onset and terminating at the end of the 31.7-second stimulation, at which point normal breathing resumed (Figure 3D). Stimulation of the amygdala via an electrode contact pair positioned slightly more lateral (contacts 2-3) induced apnea that was transient (17.1 s), with the resumption of normal 
Table 1. Patient characteristics and epilepsy history

\begin{tabular}{|c|c|c|c|c|c|c|c|}
\hline Patient no. & $\begin{array}{l}\text { Age }(y r), \\
\text { Sex }\end{array}$ & Handedness & Comorbidities & $\begin{array}{l}\text { Imaging findings (MRI, } \\
\text { PET) }\end{array}$ & Epilepsy onset (yr) & $\begin{array}{l}\text { Epilepsy duration } \\
\text { (yr) }\end{array}$ & Seizure frequency \\
\hline P447 & $3, \mathrm{~F}$ & Not established & Tuberous sclerosis & Multiple bilateral tubers & 1.1 & 1.9 & 1-4/day \\
\hline P466 & $5, M$ & L & $\begin{array}{c}\text { History of } \\
\text { myelomeningocele }\end{array}$ & Ventriculomegaly & 3.4 & 2.1 & $1 / 2$ weeks \\
\hline P381 & $5, M$ & L & None & Normal brain MRI & 1.2 & 4.6 & 1-5/day \\
\hline P422 & $9, \mathrm{~F}$ & $\mathrm{R}$ & None & $\begin{array}{l}\text { R frontal cortical } \\
\text { dysplasia }\end{array}$ & 5 & 4 & 1-11/day \\
\hline P407 & $14, \mathrm{M}$ & $\mathrm{R}$ & Anxiety and depression & $\begin{array}{l}\text { R frontotemporal } \\
\text { hypometabolism; } \\
\text { normal brain MRI }\end{array}$ & 6 & 8 & 1/week \\
\hline P412 & $17, M$ & $\mathrm{R}$ & Generalized anxiety & $\begin{array}{c}\text { Bilateral gray matter } \\
\text { heterotopia }\end{array}$ & 5 & 12 & 5-10/month \\
\hline P427 & $17, \mathrm{M}$ & Mixed & $\begin{array}{c}\text { Panhypopituitarism, } \\
\text { previous } \\
\text { craniopharyngioma } \\
\text { resection }\end{array}$ & $\begin{array}{c}\mathrm{R} \text { frontal } \\
\text { encephalomalacia }\end{array}$ & 9 & 8 & 4-20/day \\
\hline
\end{tabular}

Demographic information and epilepsy history for the patients reported in this study. Age refers to the child's age at time of electrode implantation. All patients are White and non-Hispanic. F, female; M, male; L, left; R, right; ADHD, attention-deficit/hyperactivity disorder.

breathing prior to the end of the 31.8-second stimulation (Figure 3E). In each participant, stimulation of the amygdala elicited apnea or transient apnea in multiple trials and across multiple different electrode contact pairs (sites) (Figure 3, F and G). Interestingly, stimulation of either the right or left amygdala induced apnea, indicating that inhibition of breathing is not a function lateralized to one hemisphere. Overall, these findings indicate that either the right or left amygdala can induce apnea in patients with pediatric epilepsy across a diverse cohort with varied age, epilepsy etiology, seizure type, and seizure focus (Tables 1 and 2).

Amygdala stimulation induces apnea without dyspnea. Because the amygdala plays a critical role in emotional processing and undergoes developmental changes through adolescence, we asked our subjects whether they experienced any emotional changes during stimulation. Remarkably, the patients did not report any emotional change or display any signs of emotional effects. Because even seconds without breathing can be distressing (30), we asked our patients about their lack of breathing. Strikingly, the pediatric patients were completely unaware that they had stopped breathing and did not report any shortness of breath, air hunger, desire to breathe, or display any visible signs of respiratory distress during or after the periods of stimulation-induced apnea. The lack of distress or alarm during apnea suggests that amygdala stimulation may not only inhibit breathing, but also the sensation of the need to breathe and threat of suffocation when breathing is impaired. Such striking effects were also observed in adult patients described previously (16).

The amygdala was the only stimulated forebrain structure that consistently induced apnea. In total, 342 stimulation trials were conducted at 174 stimulation sites across the 8 pediatric epilepsy research participants (Figure 4). These included 159 trials at 31 amygdala sites and 181 trials at 143 nonamygdala candidate sites (Figure 4A). Of amygdala stimulation trials, 53\% (84 of 159) produced apnea (Figure $4 \mathrm{~F})$. In stark contrast, stimulating sites outside the amygdala failed to induce apnea or transient apnea in all but 2 trials. Stimulation lateral to the amygdala and within the hippocampus, cingulate gyrus, gyrus rectus, frontal cortex, temporal cortex, and parietal cortex all failed to induce apnea or transient apnea (Figure 4, B-F). In all these structures, normal breathing persisted throughout the period of stimulation. Thus, the amygdala was the only forebrain structure that consistently induced apnea (Figure 4, A and F). The sole exception was in subject P447, where stimulation between a specific electrode contact pair $1 \mathrm{~mm}$ anterior to the amygdala caused transient apnea in 2 trials and no apnea in 3 trials. In all trials that resulted in apnea or transient apnea, breathing inhibition began within 1.9 seconds of electrical stimulation onset and normal breathing resumed within 5.5 seconds after stimulation ended (Figure 4A), suggesting that even short 5 -second stimulations were sufficient to induce apnea at sensitive sites. Together, these findings suggest that the ability of forebrain stimulation to induce central apnea is relatively specific to the amygdala. 
A

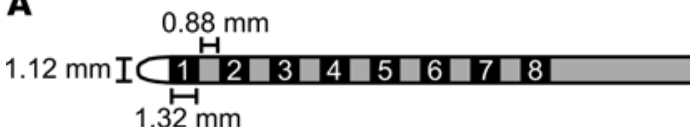

B
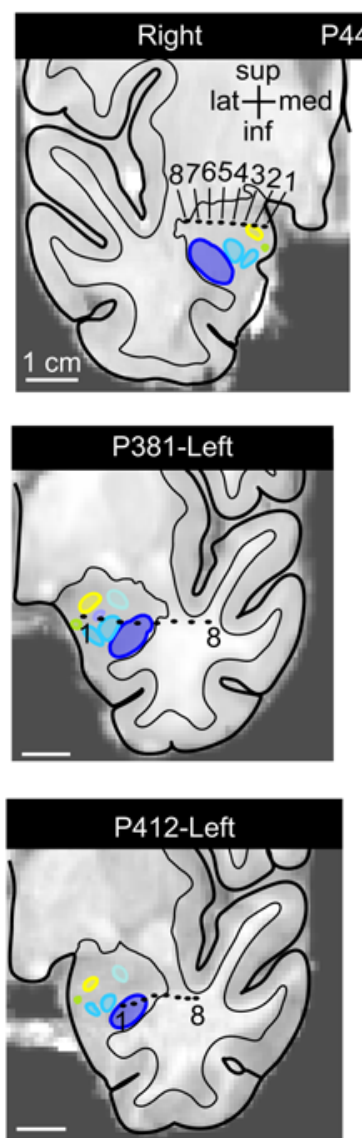
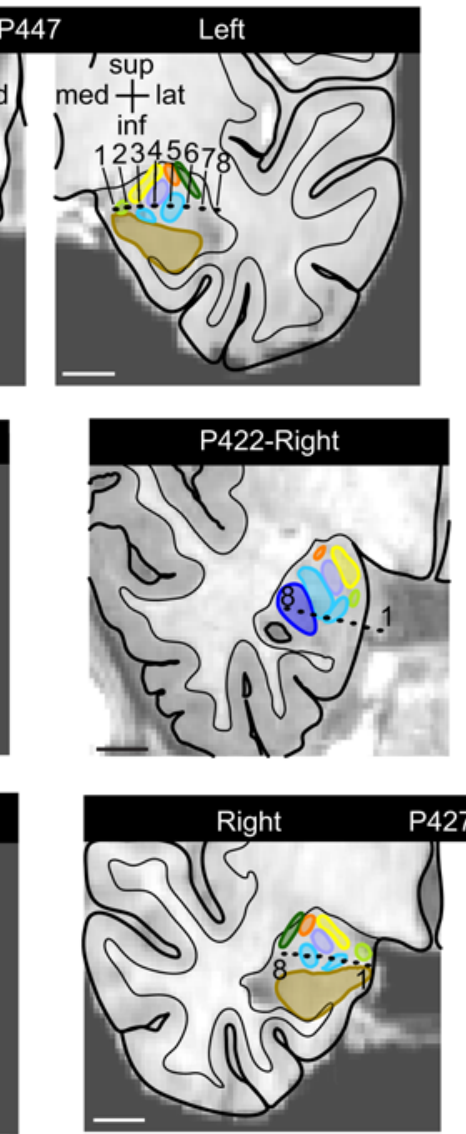

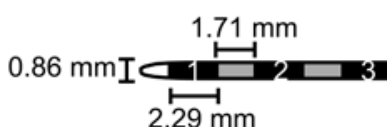

$2.29 \mathrm{~mm}$
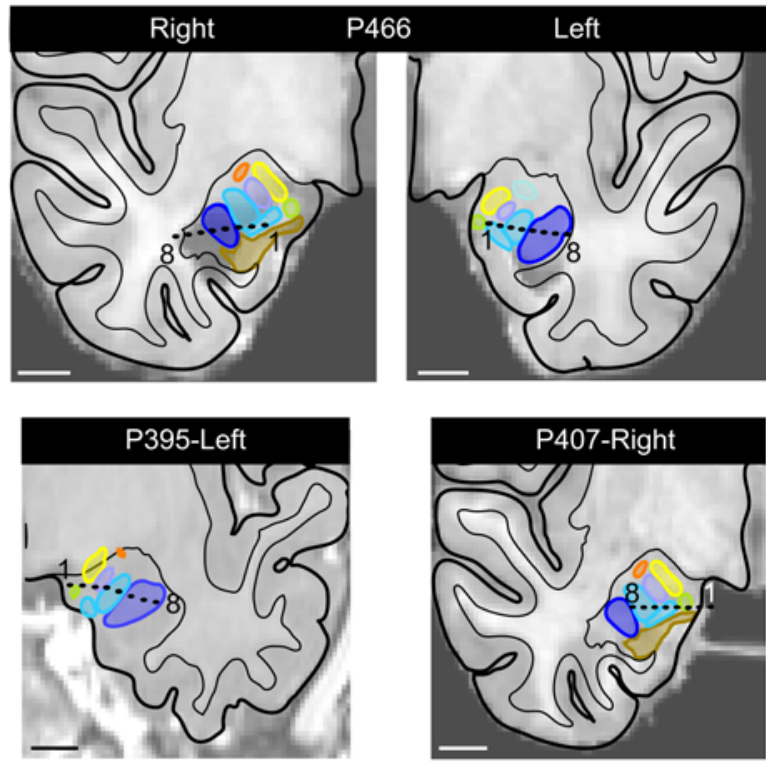

AAA $\square$ ATA $\square$ ASTA

$\square$ BL $\square$ BM $\square$ CEN

CMN $\square$ La $\square$ Hipp

Figure 1. High-density amygdala-depth electrodes were placed in 8 pediatric patients. (A) On the left, a high-density electrode with $0.88 \mathrm{~mm}$ distance between contacts measuring $1.32 \mathrm{~mm}$ long was placed in all pediatric patients except P381 (left). In P381, a high-density 8 contact electrode with 1.71 mm spacing between 2.29 - $\mathrm{mm}$-long electrode contacts was placed (right). (B) Anatomical localization of the amygdala-depth electrode contacts in the coronal plane: unilaterally in 5 patients and bilaterally in 3 patients. Each amygdala was parcellated into their individual nuclei. Numbers 1-8 specify the electrode contacts from medial to lateral. La, lateral nucleus (royal blue); BL, basolateral nucleus (light blue); BM, basomedial nucleus (lavender); CEN, central nucleus, orange; CMN, cortical and medial nuclei (yellow); ATA, amygdala transition areas (light green); ASTA, amygdalostriatal transition area (forest green); AAA, anterior amygdala area (aqua); Hipp, hippocampus (brown).

Stimulation-induced effects on breathing varied by contact location within the amygdala. Because the amygdala is composed of multiple nuclei that have distinct connectivity and functions, we hypothesized that specific locations within the amygdala may be responsible for stimulation-induced apnea. To test this hypothesis, we analyzed effects of stimulating different locations via different electrode pairs. Focal stimulation of a single nucleus or adjacent nuclei was technically feasible because high-density electrodes were used that contained closely spaced contacts (Figure 1). Due to the small size of the amygdala nuclei, adjacent electrode contacts spanned 2 nuclei in some cases. Except for the lateral nucleus $(\mathrm{La})$, this necessitated grouping adjacent nuclei together when analyzing the site-specific effects of electrical stimulation.

The highly localized nature of stimulation-induced effects on breathing was exemplified by findings in P395 (Figure 5, A-C). In this subject, apnea was induced only when electrical stimuli were delivered through contacts located in the basal nuclei (basolateral nucleus/basomedial nucleus [BL/BM]). Stimulation of the La induced transient apnea, and stimulation of the amygdala transitional area (ATA) failed to disrupt breathing (Figure 5C).

Across patients, stimulation elicited apnea at 55\% (17 of 31) of all amygdala sites. Apnea was most consistently observed when stimulation spanned the basal nuclei (Figure 5D), where 79\% of sites produced apnea that persisted throughout the duration of stimulation and $11 \%$ produced transient apnea. 
Table 2. Results of iEEG and respiratory monitoring

\begin{tabular}{|c|c|c|c|c|c|c|c|}
\hline P447 & $\begin{array}{l}\text { FOA (myoclonic } \\
\text { jerks); FOIA }\end{array}$ & $\begin{array}{l}\text { L anterior parietal } \\
\text { lobe (FOA } \\
\text { myoclonic jerks); } \\
\text { R frontal lobe } \\
\text { (FOIA) }\end{array}$ & 24 & 24 & 0 & - & - \\
\hline P466 & FOIA (clonic) & $\begin{array}{l}\text { R hemispheric- } \\
\text { multifocal, some } \\
\text { with amygdala/ } \\
\text { hippocampal } \\
\text { involvement }\end{array}$ & 12 & 10 & 9 & 24.7 & 25.4 \\
\hline P381 & FOIA & R frontoparietal & 5 & 0 & - & - & - \\
\hline P412 & FOA (clonic) & L frontal lobe & 8 & 1 & 0 & - & - \\
\hline P427 & $\begin{array}{l}\text { FOA (myoclonic } \\
\text { jerks); FOIA; GTCS }\end{array}$ & R frontal lobe & 53 & 52 & 0 & - & - \\
\hline \multicolumn{8}{|c|}{$\begin{array}{l}\text { Details of seizures observed during clinical monitoring. P381 and P395 did not comply with respiratory belts during their monitoring period. In the other } \\
\text { patients, respiratory signals during seizures could be compromised by motor artifact resulting from seizure, nonstationary status (e.g., walking, speaking) } \\
\text { or from clinical intervention. FOA, focal onset aware; FOIA, focal onset impaired awareness; GTCS, generalized tonic-clonic seizure; L, left; R, right; iEEG, } \\
\text { intracranial electroencephalography. }\end{array}$} \\
\hline
\end{tabular}

A significantly lower number of sites spanning the ATA and cortical and medial nuclei (CMN) and within the La elicited apnea when electrically stimulated, at $40 \%$ and $0 \%$ respectively (Fisher's exact test, $P=0.0011$ ). These findings suggested a region near the BL/BM may be most sensitive to stimulation in eliciting apnea.

Across subjects, amygdala stimulation-induced apnea localized to the medial aspect of the basal nuclei, ATA, and $C M N$. To examine whether a site near or within the basal nuclei may be responsible for stimulation-induced apnea, we plotted all electrode pairs within the amygdala and immediately adjacent to the amygdala, including the hippocampus (45 stimulation sites), for all subjects in a common anatomical space using Montreal Neurological Institute (MNI) coordinates (Figure 6). Electrode pairs that induced apnea were focally located in the medial portions of the amygdala and overlapped the BL/BM and ATA/CMN, consistent with the analysis using individual subject anatomy. More specifically, the electrode pairs that induced apnea appeared to localize to a more medial BL/BM region and within the ATA and CMN. Sites that induced transient apnea were lateral to the apnea-inducing sites. Sites that failed to induce apnea were located at even more lateral sites in the amygdala or in the hippocampus.

Machine-learning algorithm predicts an amygdala inhibition of respiration site. To model the amygdala region responsible for inhibition of respiration, we trained a support vector machine to predict the respiratory outcome (apnea, transient apnea, or no effect) using the MNI coordinates of 45 tested sites in the mesial temporal lobe as predictors. A machine-learning algorithm predicted areas responsible for each respiratory outcome, including an apneic area located in the medial portion of the amygdala overlapping the medial portion of BL/BM as well as ATA and CMN (Figure 7A and Supplemental Table 2; supplemental material available online with this article; https://doi.org/10.1172/ jci.insight.134852DS1). Because this area in the amygdala inhibited respiration, we refer to it as the amygdala inhibition of respiration (AIR) site (Figure 7B and Supplemental Video 1). 
A

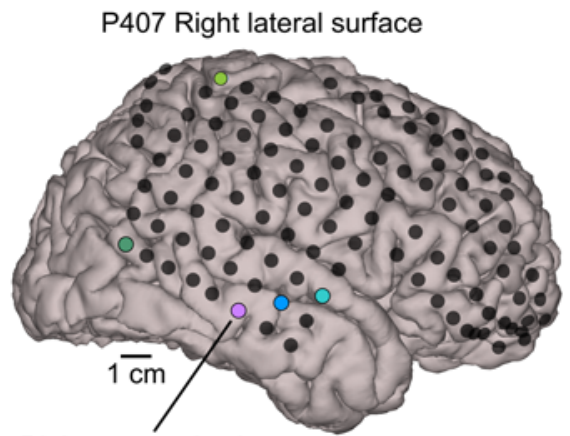

B

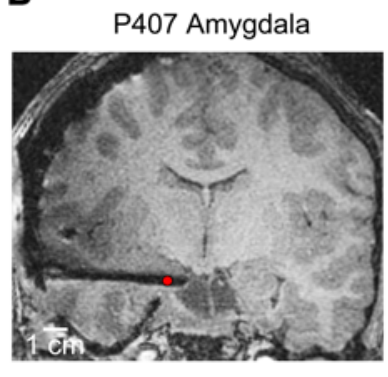

P407 Hippocampus

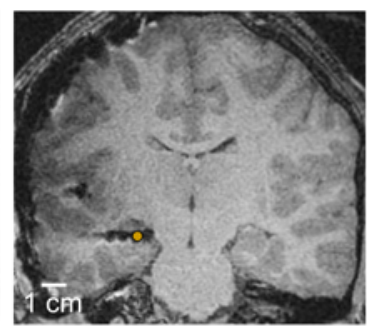

C Seizure spread to amygdala - schematic

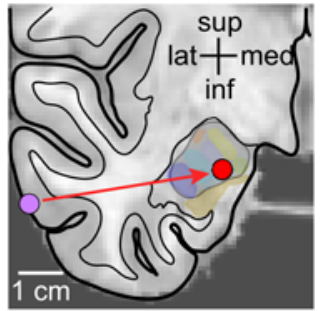

Right temporal seizure onset zone

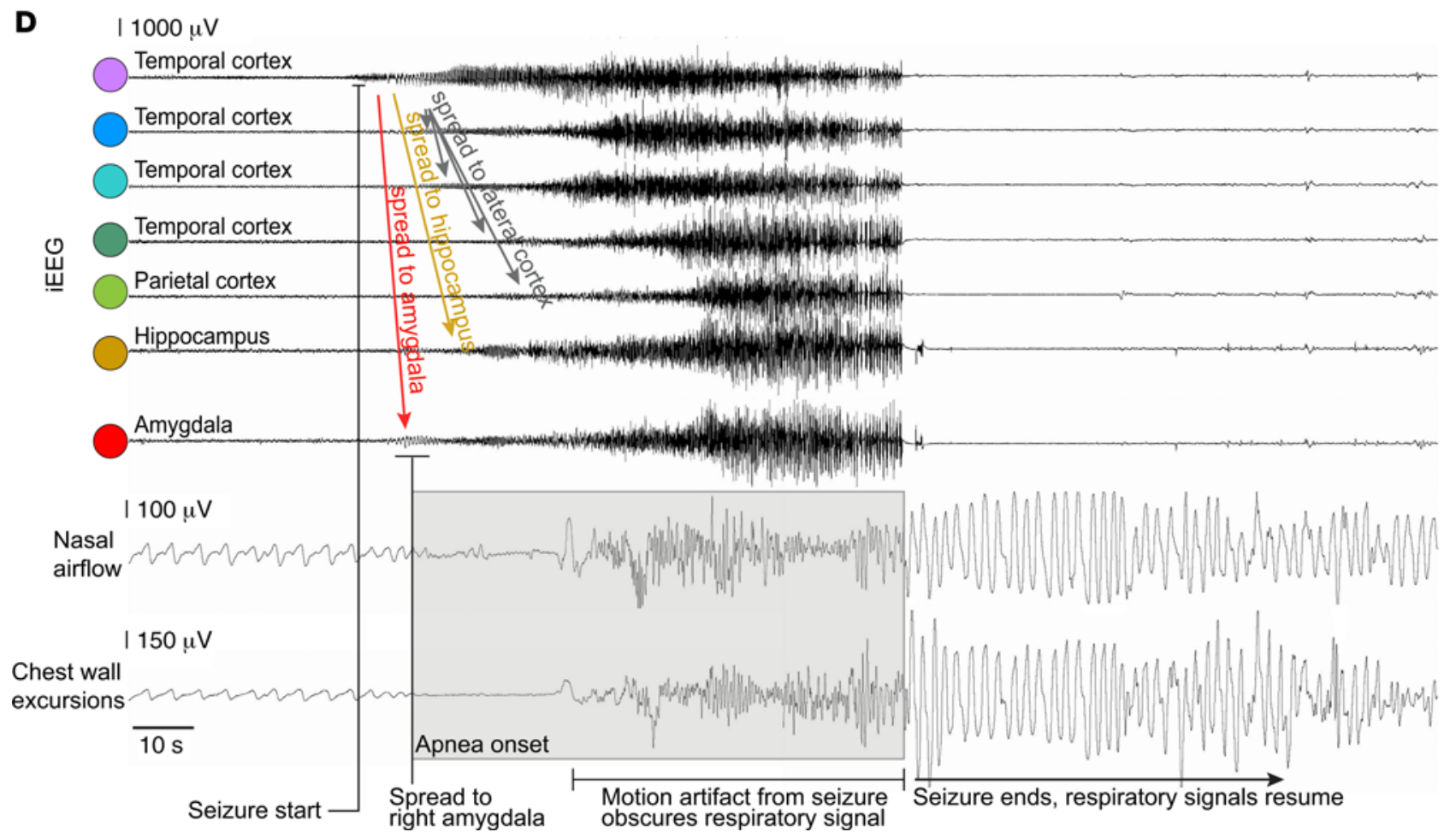

Figure 2. Apnea coincided with seizure spread to the amygdala. (A) 3D surface-rendered MR image of P407's right convexity with locations of cortical electrode contacts (black circles) in the frontal, temporal, and parietal lobes. (B) T1-weighted structural MR images showing the hippocampal and amygdala-depth electrodes and artifact produced by each electrode contact. (C) Schematic illustrating seizure spread from the lateral temporal cortex (purple circle, seizure onset) to the amygdala (red circle). (D) iEEG of seizure originating in lateral temporal cortex (purple circle in $\mathbf{A}$ ) and spreading quickly to the amygdala (red circle in B). Breathing is normal until seizure activity spreads to the amygdala, which coincides with apnea and loss of normal breathing (inspiration is plotted up). The seizure spreads 10 seconds later to multiple other recording contacts in the hippocampus, temporal cortex, and parietal cortex, as colored in $\mathbf{A}$ and $\mathbf{B}$, and then generalizes, at which point motion artifact obscures the respiratory signal. Normal breathing is observed after seizure end.

\section{Discussion}

In this report we make use of the rare opportunity to study pediatric patients implanted with intracranial electrodes undergoing continuous respiratory and seizure monitoring to directly examine how epileptic seizures and electrical stimulation of the amygdala alters breathing in children. Thus, we were able to test a number of important questions about the amygdala in seizure-induced apnea and SUDEP in children.

First, we explored in children across a range of ages whether the amygdala plays a role in seizure-induced apnea, as in adults $(16,23)$, and whether amygdala stimulation might be sufficient to inhibit respiration. The amygdala undergoes functional and structural connectivity changes throughout adolescence (24-26), raising the possibility that effects in adults might not occur in children. Nevertheless, here we observed seizure-induced apnea in 1 patient who was 5 years old and in another who was 14 years old; 
A

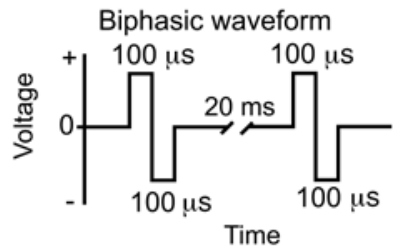

B

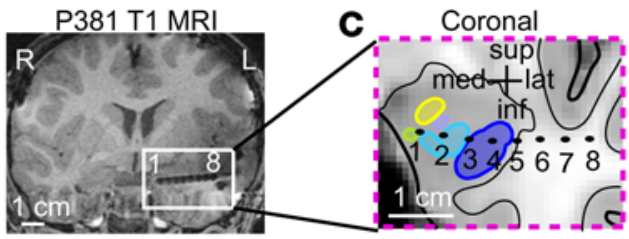

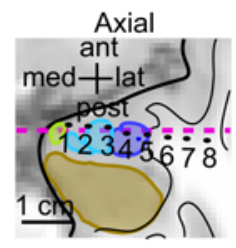

D

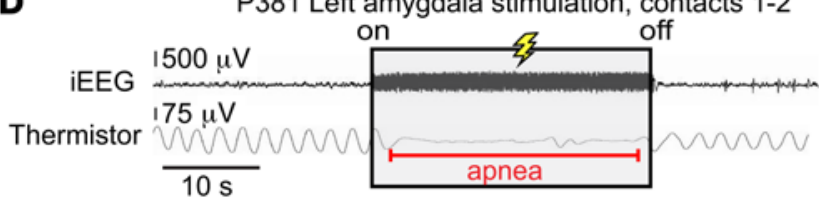

$\mathbf{F}$

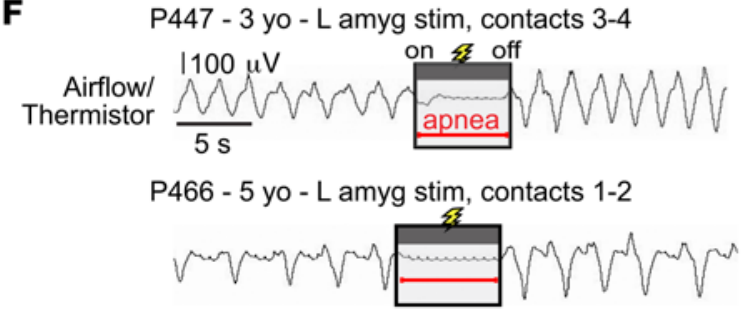

P422 - 9 yo - R amyg stim, contacts 4-5

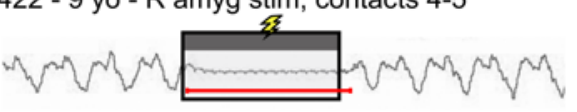

P407 - 14 yo - R amyg stim, contacts 4-5

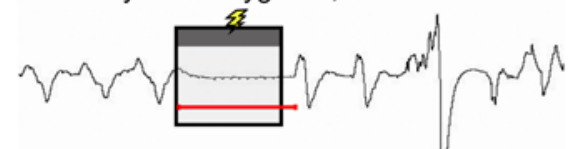

P427 - 17 yo - L amyg stim, contacts 3-4

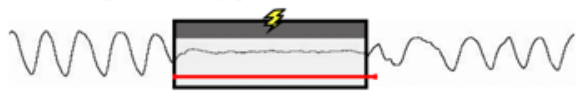

E

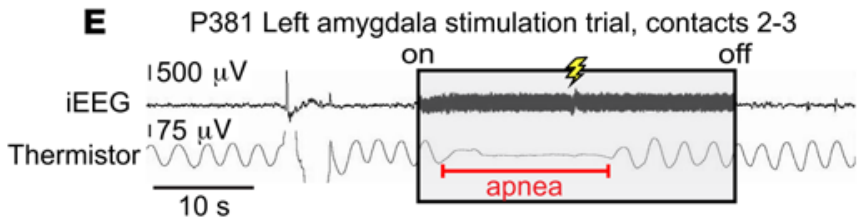

P447 - 3 yo - R amyg stim, contacts 2-3

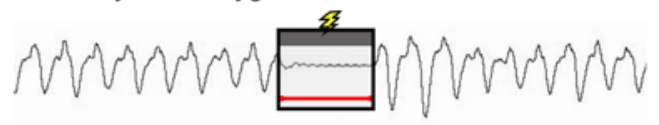

P466 - 5 yo - R amyg stim, contacts $1-2$

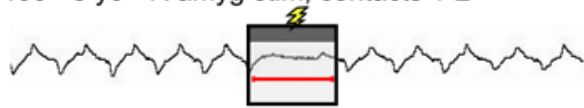

P395 - 13 yo - L amyg stim, contacts 3-4

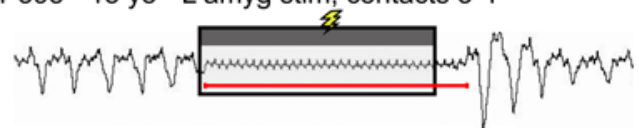

P412 - 17 yo - L amyg stim, contacts 1-2

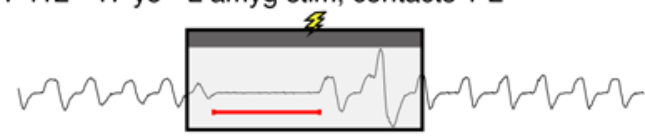

P427 -17 yo - R amyg stim, contacts 2-3

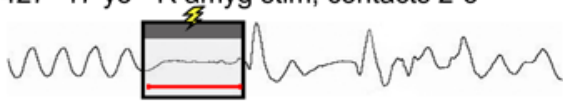

G

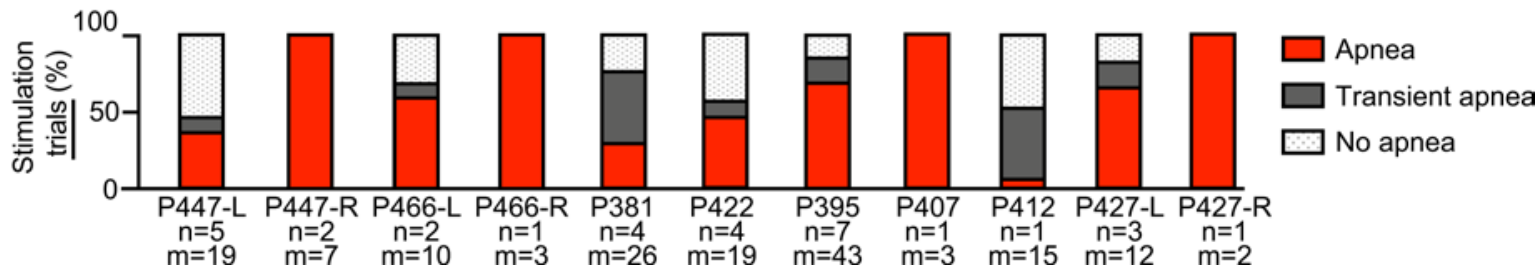

Figure 3. Electrical stimulation of the amygdala induced varying degrees of apnea in all 8 patients with pediatric epilepsy. (A) Focal electrical stimulation was delivered using a bipolar biphasic square waveform, 200- $\mu$ s pulse width, $50-\mathrm{Hz}$ frequency, and constant voltage (see Methods). (B) Coronal T1-weighted structural MR image showing the left amygdala-depth electrode in subject P381. Artifacts produced by each contact from most medial to lateral are labeled 1 to 8 , respectively. (C) Location of electrode contacts superimposed upon an anatomic diagram of P381's left temporal lobe with amygdala nuclei, hippocampus, and surrounding areas in coronal and axial planes. The dotted magenta line within the axial view represents coronal plane outlined with same dotted line. Amygdala nuclei defined as in Figure 1B. (D) Amygdala stimulation (contacts 1 and 2) in P381 resulted in apnea that was almost immediate in onset and lasted the duration of stimulation. iEEG signal shown on top and respiratory signal from oral/nasal thermistor shown below (inspiration plotted up; duration of stimulation depicted by shaded gray box; duration of apnea shown by horizontal red line; conventions remain the same for $\mathbf{E}$ and $\mathbf{F}$ ). (E) Stimulation of more lateral sites in the amygdala (contacts 2 and 3) in P381 resulted in transient apnea: apnea was almost immediate in onset ( $<2 \mathrm{~s}$ from stimulation onset) but did not last the entire duration of stimulation, and normal breathing resumed despite continued stimulation. (F) Amygdala stimulation elicited apnea or transient apnea in the 7 other pediatric patients. Respiratory traces shown from oral/nasal thermistor or nasal pressure transducer. (C) Bar graph illustrating the percentage of trials eliciting each of the 3 types of breathing effects (apnea, red; transient apnea, dark gray; no apnea, dot shading) for all 8 patients and 11 amygdalae. Beneath each bar are the number of sites stimulated $(n)$ and number of trials $(m)$. In total, 159 stimulation trials within 31 different sites induced apnea to varying degrees.

and in both cases, apnea occurred when seizures spread to the amygdala. Similarly, electrical stimulation of the amygdala consistently inhibited respiration in all of our patients, ranging in ages from 3 to 17 years. Together, these observations indicate that developmental changes in amygdala connectivity during childhood and adolescence are not required for the amygdala to inhibit respiration. Further, they indicate that 
A

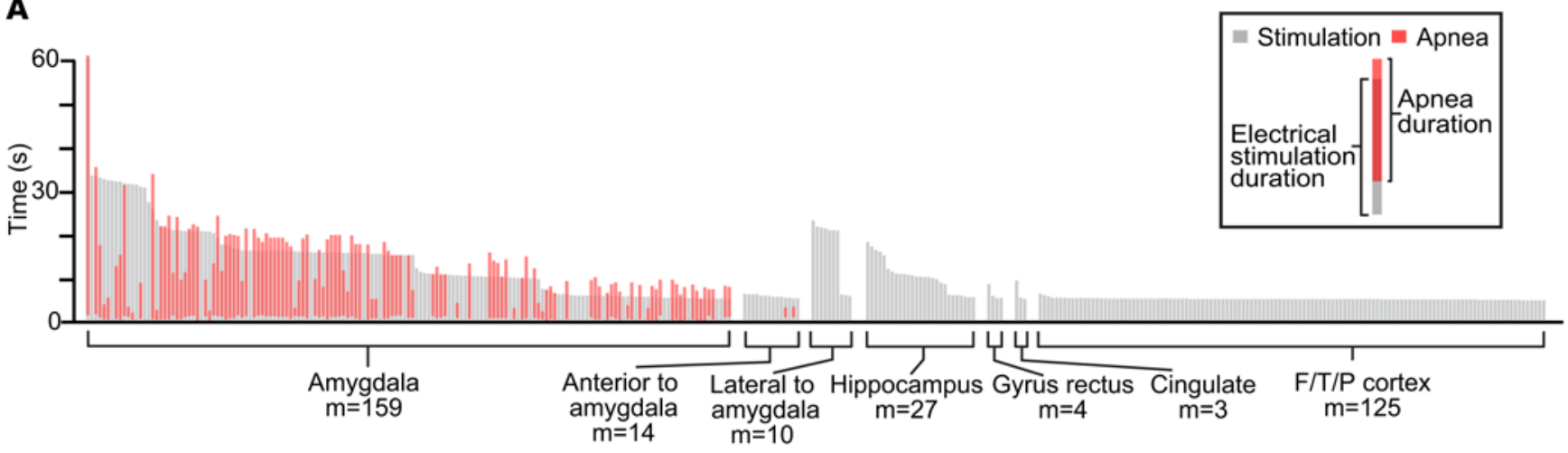

B Lateral to amygdala - P381 Contacts 5-6
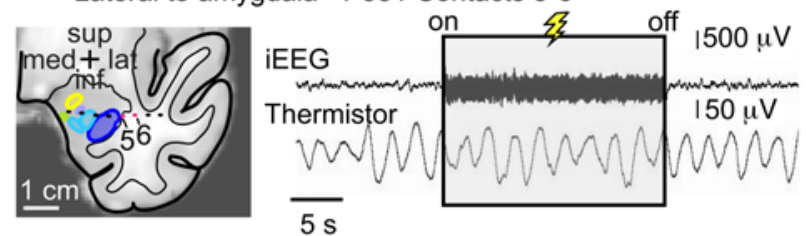

C Hippocampus - P395 Contacts 2-3
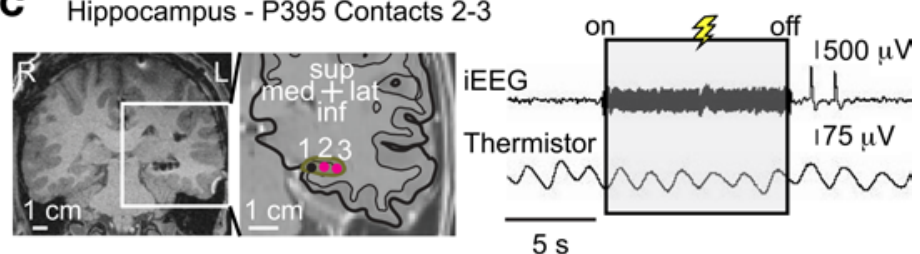

D

Cingulate gyrus - P395
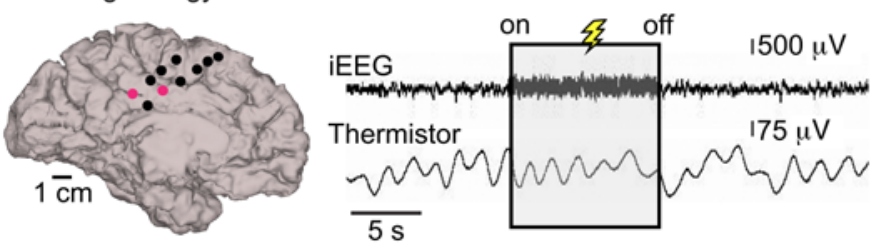

E Frontal cortex - P395
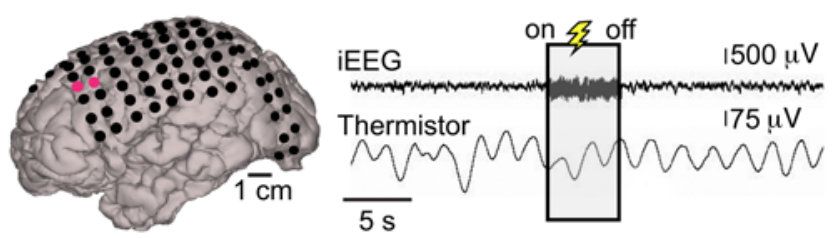

$\mathbf{F}$
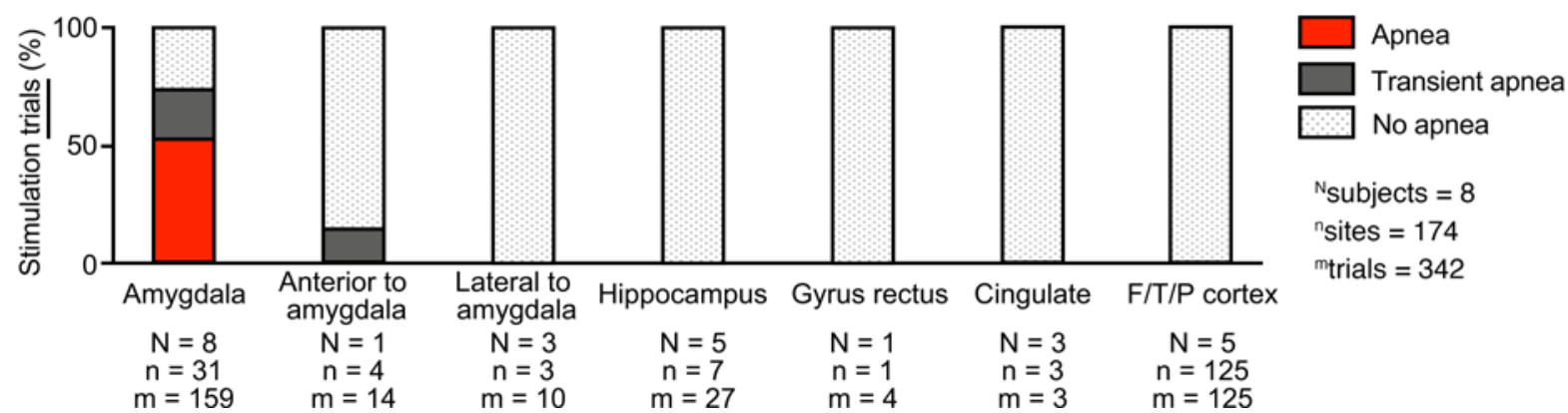

${ }^{\mathrm{N}}$ subjects $=8$

"sites $=174$

mtrials $=342$

Figure 4. Stimulation-induced apnea was relatively specific to the amygdala and not seen with stimulation of any other forebrain site. (A) Plot illustrating durations of stimulation and durations of apnea for each trial, shown by gray and red vertical lines, respectively. All trials of stimulation were at least 5 seconds in duration. Stimulation always induced apnea within 1.9 seconds after onset. Apnea was only observed with stimulation of the amygdala. (B) Location of electrode contacts superimposed upon an anatomic diagram of P381's left temporal lobe with amygdala nuclei and surrounding areas in coronal plane. Amygdala nuclei defined as in Figure 1B. Stimulation outside and immediately lateral to the amygdala (contacts 5 and 6, pink) failed to elicit apnea. (C) Coronal T1-weighted structural MR image showing the left hippocampus depth electrode in subject P395. Electrode contacts from most medial to lateral are labeled 1-3. Location of electrode contacts superimposed upon an anatomic diagram of the left temporal lobe with hippocampus and surrounding areas in coronal plane. Stimulation of the hippocampus (contacts 2 and 3, pink) failed to elicit apnea. (D) 3D surface-rendered MR image of P395's left medial hemisphere with superimposed electrode contacts in P395. Stimulation of the cingulate gyrus (pink circles) failed to elicit apnea. (E) 3D surface-rendered MR image of P395's left convexity with superimposed electrode contacts. Stimulation of the left frontal cortex (pink circles) failed to elicit apnea. (F) Bar graph illustrating the percentage of trials eliciting each of the 3 types of breathing effects (apnea, red; transient apnea, dark gray; no apnea, dot shading) for all patients. Beneath each bar are the number of subjects $(N)$, number of sites stimulated $(n)$, and number of trials $(m)$. Except for 1 electrode pair immediately anterior to the amygdala where stimulation led to transient apnea, stimulation at all other sites and all other trials failed to induce any measurable changes in breathing.

a connection between the amygdala and brainstem respiratory network is established in humans prior to age 3 , either in early postnatal development or in utero. This functional connection likely underlies at least some cases of seizure-induced central apnea in children as well as adults. Moreover, although it remains to be proven, we speculate that this connection may have important implications for SUDEP and possibly 
A
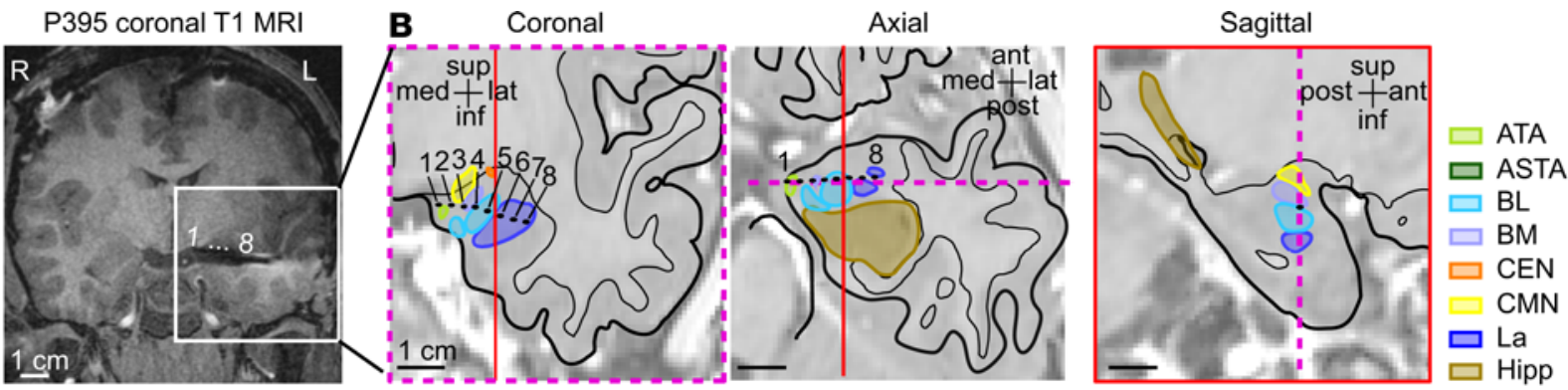

C

ATA, contacts 1-2

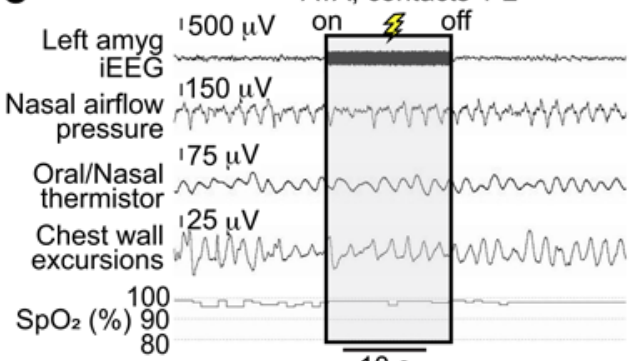

$\mathrm{BL}$, contacts 4-5

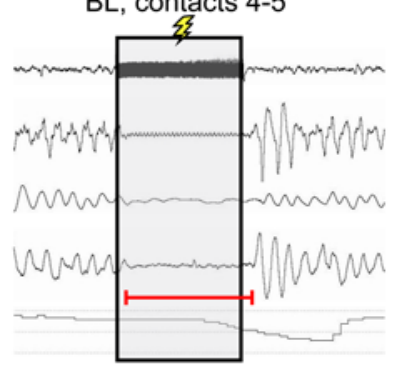

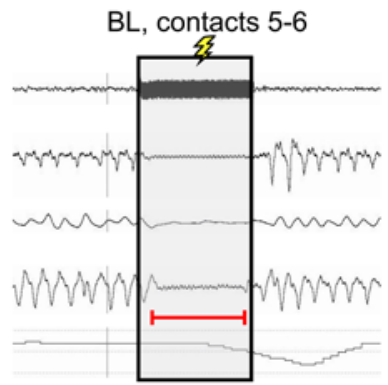

BL/BM, contacts 2-3

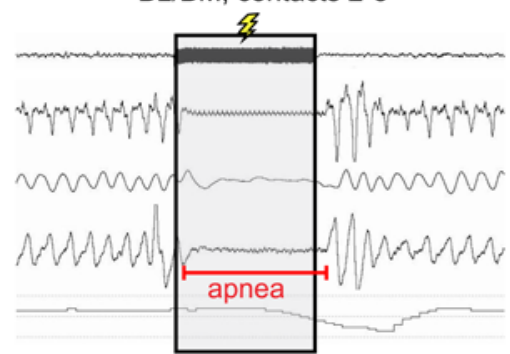

La, contacts 6-7

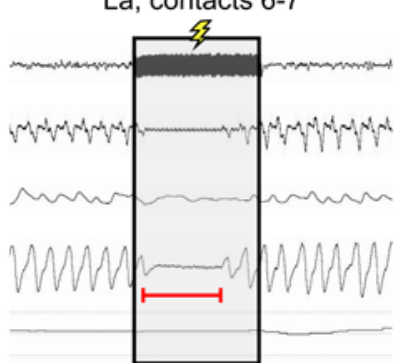

BL/BM, contacts 3-4

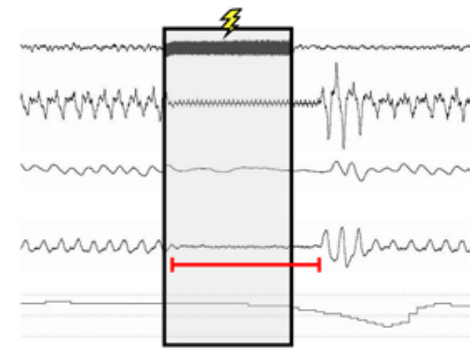

La, contacts 7-8

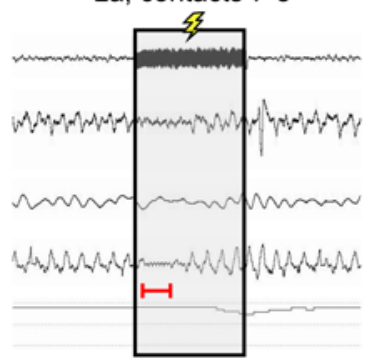

D

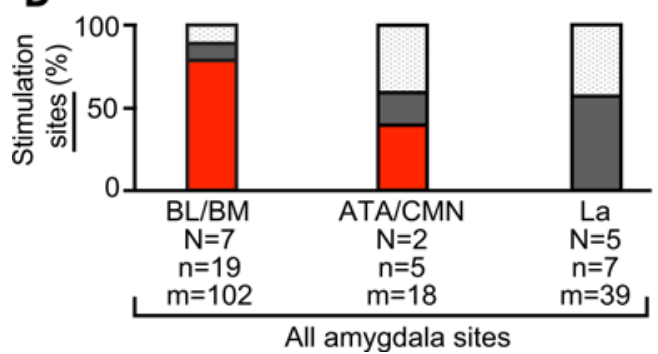

Apnea

Transient apnea

No apnea

Figure 5. Stimulation-induced effects on breathing varied by contact location in the amygdala. (A) Coronal T1-weighted structural MR image showing the left amygdala-depth electrode in subject P395. Electrode contacts from most medial to lateral are labeled 1 to 8 , respectively. (B) The location of electrode contacts superimposed upon anatomic diagrams of P395's left temporal lobe with amygdala nuclei, hippocampus, and surrounding areas in coronal, axial and sagittal planes. The dotted magenta line within the axial and sagittal view represents coronal plane outlined with the same dotted line. The red line in the axial and coronal view represents the sagittal plane outlined with the same red line. Amygdala nuclei defined as in Figure 1B. (C) Stimulation of bipolar contacts 1-8 from medial to lateral within the amygdala nuclei elicited different effects on breathing. Apnea was observed with stimulation of the basal nuclei (BL/BM) and only transient apnea was observed with stimulation of the lateral amygdala. No apnea was observed with ATA stimulation. Oxygen desaturation corresponded with the length of apnea and transient apnea at each site. (D) Bar graph depicting across-subject respiratory effects in the 3 grouped amygdala nuclei for which stimulation elicited effects on breathing. Within the BL/BM, $79 \%$ of stimulated electrode contact pairs were characterized by apnea, and $11 \%$ were characterized by transient apnea. Fewer sites in the ATA/CMN showed breathing disruption (40\% apnea, $20 \%$ transient apnea). Transient apnea (57\% of sites) or no apnea occurred with electrical stimulation of the La. Omnibus Fisher's exact test showed significant association between the 3 nuclei and the 3 respiratory outcomes $(P=0.00137)$. Post hoc planned comparisons showed significant association between sites in BL/BM and apnea (BL/BM versus other areas, and apnea versus other respiratory outcome, Fisher's exact test, $P=0.0011)$. Oxygen desaturation nadir occurred after amygdala stimulation because oxygen saturation was measured with a pulse oximeter on the extremities resulting in a 15- to 20-second delay.

sudden infant death syndrome (SIDS) and sudden unexplained death in childhood (SUDC), conditions that parallel SUDEP (31-33).

Despite disrupted breathing, it was striking that no child ever reported shortness of breath or dyspnea and no child displayed any visible signs of respiratory distress, emotional distress, or arousal. 

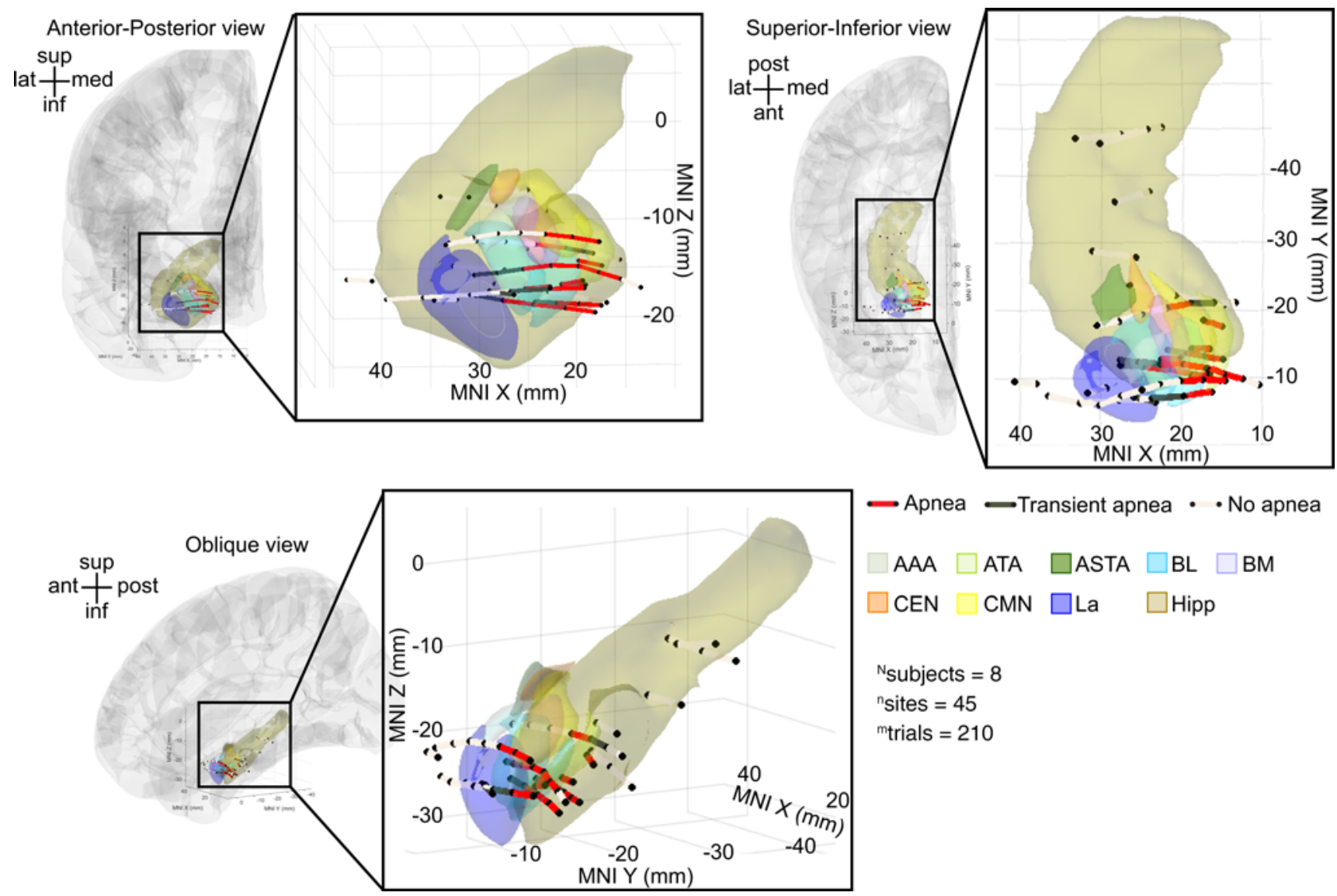

-Apnea -Transient apnea • • No apnea

AAA $\square$ ATA $\square$ ASTA $\square$ BL $\square$ BM

$\square$ CEN $\square$ CMN $\square$ La $\square$ Hipp

${ }^{\mathrm{N}}$ subjects $=8$

"sites $=45$

mtrials $=210$

Figure 6. Across-subject analysis localized amygdala stimulation-induced apnea to the medial aspect of BL/BM and the ATA/CMN. Anterior-posterior superior-inferior, and oblique views of all stimulated electrode pairs in the amygdala and hippocampus across subjects plotted in a common coordinate system (MNI). Electrode contact pairs that produced apnea were located in the medial BL/BM and ATA/CMN. Electrode contact pairs that produced transient apnea were typically located just lateral or adjacent to this medial BL/BM region. Electrode contact pairs that failed to induce apnea were located in La, outside the amygdala, or in the hippocampus. Electrode pairs that induced apnea are denoted by dark red lines; those that produced transient apnea are depicted in dark gray lines; sites that did not induce apnea are depicted in light gray. For clarity, electrode sites on the lateral convexity, in the cingulate gyrus, and gyrus rectus are not shown; no sites omitted from this figure demonstrated effects of electrical stimulation on breathing. See Supplemental Table 1 for a list of MNI coordinates and the respiratory effect for each contact pair. Nuclei are color-coded with the same convention as in Figure 1B. Electrode contacts may appear outside of the template brain due to anatomical variation across subjects relative to the MNI coordinate system. All electrode contacts were plotted in the right hemisphere for simplicity, because no differences were observed between right and left amygdala stimulation.

These findings were consistent with previous observations in adults (16) and were remarkably similar to the lack of apparent struggle or arousal observed in nonhuman primates upon amygdala stimulation (34) and in patients who died from SUDEP (9). Thus, seizure spread to the amygdala may suppress emotional distress and air hunger at the same time that it inhibits breathing. Supporting a coordinated control of arousal and breathing by the amygdala, $\mathrm{CO}_{2}$-evoked arousal and respiration were increased in patients with bilateral amygdala lesions (35). An absence of dyspnea and/or emotional alarm could be critical during seizures, as it could interfere with a patient's ability to maintain and protect their airway, especially if face down in bed, which could be catastrophic if they are alone and no one is nearby to intervene (9).

Because epileptic seizures can induce plasticity and alter brain connectivity (36), it is conceivable that different epilepsy types may induce different patterns of brain connectivity and enhance or reduce the amygdala's ability to suppress breathing. Therefore, we asked whether the amygdala's effects on breathing would be observed across epilepsy types. Interestingly, amygdala stimulation consistently induced apnea across a spectrum of epilepsies, varying in seizure foci and pattern of spread. Thus, apnea did not depend on epilepsy type. Moreover, in a previous study, amygdala stimulation induced apnea even in a patient without epilepsy (16). These observations indicate that the connection between the amygdala and brainstem respiratory networks is intrinsic, robust, and independent of epilepsy. However, they do 
A

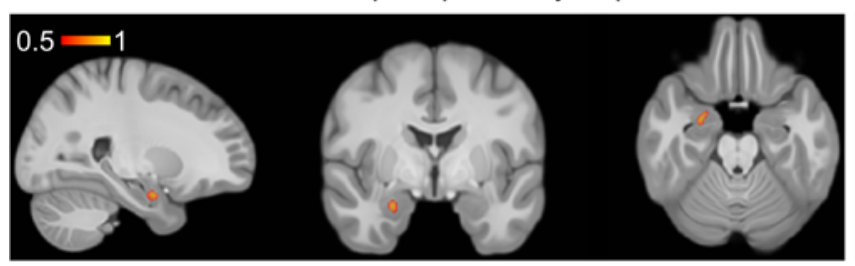

Apnea probability map

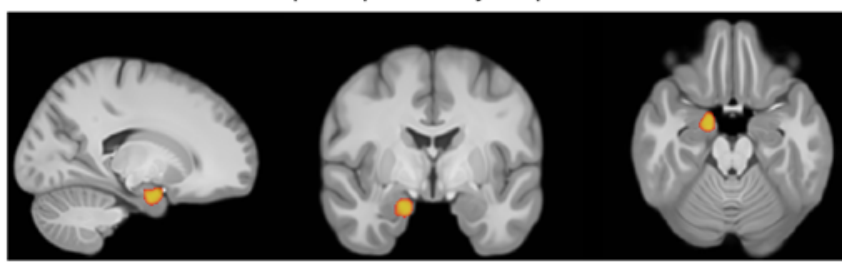

Amygdala

Hippocampus

AIR site
B

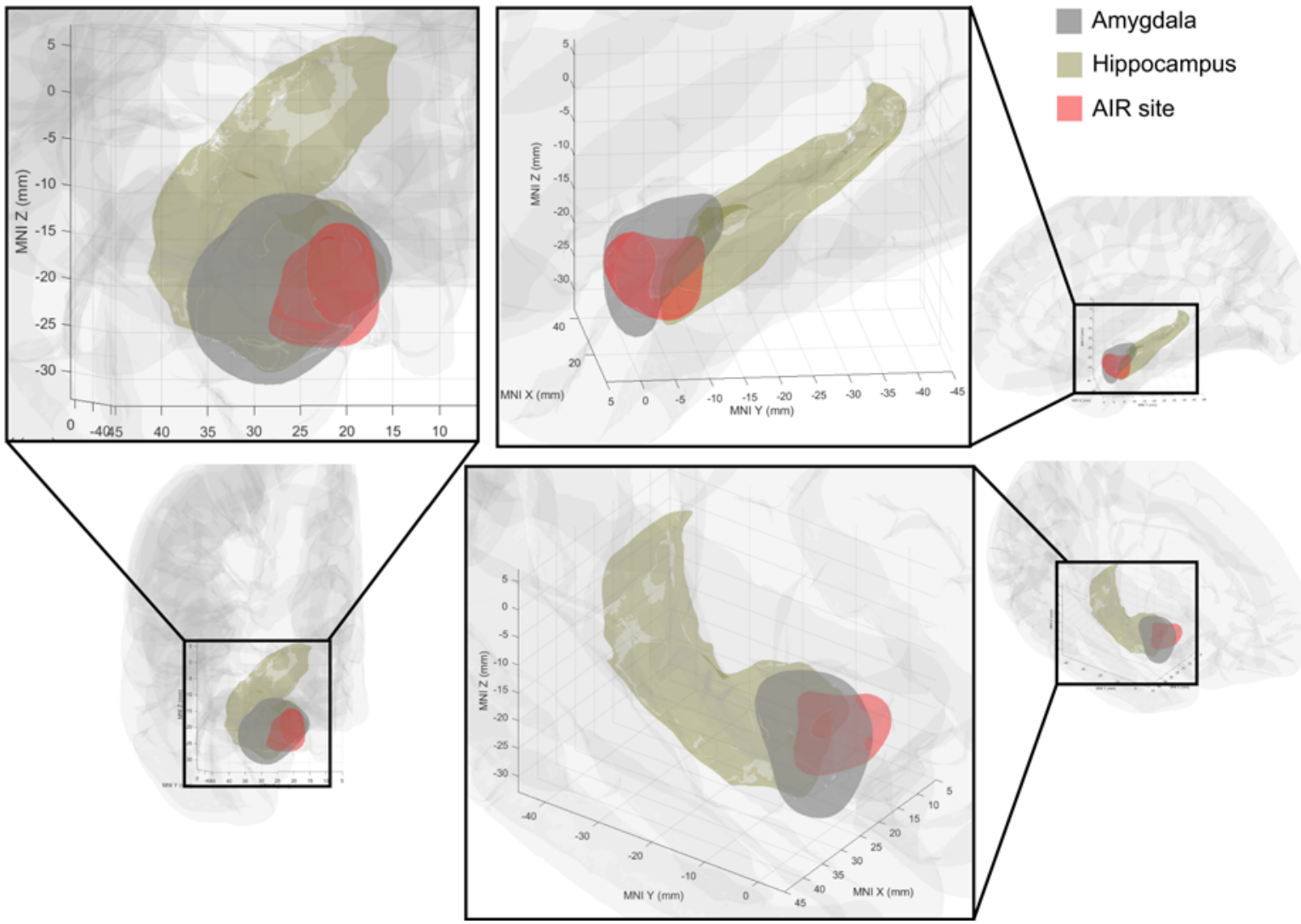

Figure 7. Machine-learning algorithm localizes an amygdala inhibition of respiration site. (A) Probability map of transient apnea (left) and apnea (right) resulting from support vector machine classification of respiratory effects predicted from MNI coordinates of 45 tested mesial temporal electrode contact pairs. (B) The amygdala inhibition of respiration (AIR) site (red) predicts apnea based on the results of A, overlaid on amygdala (gray, FSL) and hippocampus (brown, FreeSurfer). Probability map is plotted in right hemisphere only for simplicity, because no differences were observed between right and left amygdala stimulation.

not rule out the possibility that over time repeated seizures might strengthen this circuit and increase the likelihood or severity of seizure-induced apnea.

Breathing is essential for life, and thus the ability of the amygdala to inhibit breathing might be surprising. However, it has long been recognized that forebrain structures in humans can control breathing, and in many cases this control involves emotion, such as in speech, laughing, and crying (18-22). Emotion is thought to depend at least in part on the amygdala. The amygdala is critical for mediating defensive responses to a variety of threats, including freezing and fight-and-flight responses $(37,38)$, where altering respiration can be crucial for survival. Thus, a pathway by which the amygdala influences breathing might be advantageous from an evolutionary perspective.

In addition to the amygdala, other forebrain sites have been implicated in breathing control, stimulation-evoked apnea, and apnea during seizures $(15-17,19,23,29,39)$. Thus, it is conceivable that stimulation of other forebrain sites might also induce apnea. Therefore, we investigated whether apnea was relatively specific to amygdala stimulation,or if it would be seen with other sites. Interestingly, however, stimulating numerous sites outside the amygdala, including the hippocampus and cingulate gyrus, failed to induce any effect on breathing in this cohort. The only exception was a site immediately anterior to the 
amygdala in P447, which elicited transient apnea in 2 trials likely due to current spread from stimulation within immediate proximity to the amygdala.

The amygdala is composed of multiple nuclei with different functions. Therefore, we asked if specific regions of the amygdala mediate apnea. Stimulating a circumscribed zone in the medial amygdala consistently produced apnea. This site, which we call the AIR site, overlaps the very medial aspect of the basal nuclei (BL/BM), CMN, and ATA and also likely includes intercalated (ITC) neurons, a cluster of inhibitory neurons lying between the BL/BM (40-42). From animal studies, several projections from neurons within the AIR site might mediate apnea. First, BM and ITC neurons project directly to the parabrachial nucleus, a part of the brainstem central pattern generator (43-50) controlling timing and phase transitions of the respiratory cycle via the pre-Bötzinger complex, a key driver of inspiration and respiratory rhythm (51-53). A second pathway by which neurons in the AIR site might affect breathing is via the central nucleus (CEN); the BL, $\mathrm{BM}$, medial nucleus, and ITC project to the $\operatorname{CEN}(27,49,54,55)$, which in turn also projects to sites in the breathing central pattern generator (43-50), including the pre-Bötzinger complex (51-53). A third pathway by which breathing may be affected is via projections from the medial nucleus to both the hypothalamus and bed nucleus of the stria terminalis $(55,56)$, which both in turn project to the pre-Bötzinger complex $(48)$. As mentioned above, the absence of dyspnea and emotional alarm during the apneic episodes was surprising and suggests that dyspnea and arousal might also be suppressed by electrical stimulation. An inhibitory connection to midbrain neurons sensitive to $\mathrm{CO}_{2} / \mathrm{pH}$ might suppress dyspnea and arousal (57), although connections between these neurons and the AIR site are not clear. Alternatively, the parabrachial nucleus, which receives direct projections from AIR site neurons, promotes arousal when $\mathrm{CO}_{2}$ concentrations rise, and might be ideally positioned to affect dyspnea and arousal, as well as breathing (58). Finally, it is conceivable that AIR site projections to cortical, limbic, and paralimbic sites might also suppress dyspnea (59).

There are limitations to the methods required by these studies that deserve consideration. With electrical stimulation, we cannot precisely target specific neurons and circuits. Using finite element modeling of bipolar stimulation, with the parameters we used in this study, current spread is localized to a small discrete region around the bipolar electrode contacts (60). Regardless, we cannot exclude that apnea was induced from current spread to immediately nearby sites or white matter tracts; nor is it known that the electrical stimulation that elicits apnea is activating, inhibiting, or a combination of both. Further, we were unable to test whether stimulation of sites within the CEN or AAA could elicit apnea because electrode contacts were not located here. Focal stimulation of these small nuclei as well as other forebrain sites will require further study. Although high-resolution MRI and CT allow high-quality imaging of individual brain structures and precise location of electrode contacts, the human amygdala atlas (28) used to define the nuclei was derived from a study of normal human subjects and thus may differ from our epilepsy cohort. Supporting the use of this atlas, no structural abnormalities were detected by T1- and T2-weighted imaging in any amygdala of the patients reported here, suggesting that amygdala anatomy was likely similar to the normal population. Nevertheless, it remains possible that chronic epilepsy might induce functional or structural connectivity changes in the amygdala over time. And finally, because unilateral amygdala seizures without contralateral amygdala involvement can elicit apnea (P466), seizures in or stimulation of only 1 amygdala appears sufficient to inhibit breathing. Nevertheless, we cannot rule out that both amygdalae are required for breathing inhibition.

Importantly, localizing brain sites responsible for seizure-induced loss of breathing in patients with epilepsy could be key to preventing SUDEP. Novel imaging strategies might reveal abnormalities within the AIR site or differences in connectivity between the AIR site and brainstem respiratory sites. Such efforts could help predict who might be at the greatest risk for seizure-induced apnea and SUDEP, for example, if it were found that connections between the amygdala and respiratory sites were abnormally strong in specific individuals. Moreover, localizing the AIR site provides a potential therapeutic target. Developing methods to inhibit the AIR site and its connection to brainstem respiratory networks via drug based or surgical strategies may limit apnea during seizures and thus help prevent SUDEP.

\section{Methods}

Patients. Eight pediatric patients with medically intractable epilepsy were studied while undergoing iEEG during a 2- to 3-week monitoring period for seizure focus localization (age range, 3-17 years old; 2 females; see Table 1 for patient characteristics and epilepsy history). Intracranial electrodes (Ad-Tech Medical Instrument Corporation) were implanted at sites determined by the multidisciplinary epilepsy team at the University of Iowa. During the monitoring period, antiepileptic drugs were tapered to allow 
seizures to occur to identify seizure foci; antiepileptic drugs were restarted prior to electrical stimulation used in the current study.

Data acquisition, imaging, and electrode localization. iEEG data were acquired using a Nihon Kohden JE-120 256 channel amplifier and analyzed using NeuroWorkbench software. Patients were monitored at the University of Iowa Stead Family Children's Hospital. Experimental recordings did not interfere with the collection of clinically relevant data. Seizure foci and timing of seizure onset and spread were determined by analysis of iEEG by a pediatric epileptologist with independent assessment by a pediatric epilepsy neurosurgeon. Electrode localization was performed using MR and CT imaging using techniques previously reported $(16,61)$. For each patient, whole-brain high-resolution T1-weighted structural 3T MRI scans (resolution and slice thickness, $\leq 1.0 \mathrm{~mm}$ ) were obtained before and after electrode implantation. CT and MR structural scans after implantation were linearly coregistered to MR scans before implantation using the FLIRT module of FSL (62). Images were corrected for displacement and deformation from surgery using nonlinear thin-plate spline warping using manually selected control points (63). Finally, FreeSurfer was used to identify the cortical surface and white-gray boundaries within T1 images before implantation, which were corrected manually based on visual inspection where necessary (64). High-density depth electrodes containing closely spaced contacts (Figure 1A) were placed in all 8 pediatric patients. In 5 patients, only 1 amygdala was implanted, and in 3 patients, both amygdalae were implanted. In total, 11 amygdalae contained depth electrodes across 8 subjects (Figure 1B).

Amygdala nuclei parcellation. Amygdala nuclei were delineated and parcellated using the CIT168 human brain template (28). This was conducted using a high-precision nonlinear volumetric coregistration of preoperative structural T1 and T2 imaging onto the template brain. Eight amygdala nuclei were delineated, and the structural relationships between electrode contacts and nuclei were determined from the projected contact locations within the preoperative brain images. For more clarity and simplicity, as described in Tyszka and Pauli (28), the BL as labeled in this study includes the dorsal, intermediate, and ventral/ paralaminar subnuclei of the BL. The CIT168 atlas is derived from in vivo imaging in 168 subjects and thus provides a much finer parcellation of the amygdala with more accurate external boundary definition than current ex vivo histology-based atlases or other nuclei parcellations from other sources. Moreover, amygdala volume (both right and left) does not significantly vary across age, as observed in a study of 166 participants from 5 to 30 years old (26), thus supporting the use of this anatomic atlas in children.

Electrical stimulation. Electrical stimulation was conducted at the bedside while the subjects were awake and in a relaxed state (16). Electrical stimulation for functional brain mapping is a well-established and safe method used routinely for clinical and research purposes in neurosurgery patients $(16,65)$. Bipolar biphasic stimulation was conducted with parameters set at $200-\mu$ s pulse width, a frequency of $50 \mathrm{~Hz}$, and constant voltage using a Grass SD9 stimulator. Pairs of adjacent electrode contacts (sites) were stimulated. A dose-response curve for each subject was obtained by increasing stimulation voltage beginning at 2.5 $\mathrm{V}$ until breathing was affected up to a maximum of $15 \mathrm{~V}$ (the typical threshold for motor movement with stimulation of the motor cortex in these subjects). No afterdischarges occurred during experimental stimulation trials. With increasing voltage during the establishment of the dose-response curve, apnea was an all or none effect. Apnea occurred at $10 \mathrm{~V}$ in all patients except P381 and P412, where apnea was observed with $15 \mathrm{~V}$, consistent with previous findings (16). Stimulation duration was varied between patients based on the proximity of the stimulation site to the patient's seizure focus (range, 5-60 seconds). Sites in close proximity to the seizure focus were stimulated for shorter durations. To allow for recovery between trials, we maintained an interval of 1.5-2.5 minutes from the end of one trial to the beginning of the next. No subjects showed evidence of, or complained of, any pain or discomfort during stimulation. Patients and their parents/guardians were blinded to the delivery of the electrical stimulation.

Respiratory measurements. To record breathing, patients wore a chest/abdominal plethysmography belt (ProTech zRIP) during their 2- to 3-week monitoring period for seizure focus localization. Oral and nasal airflow were additionally measured with a BiNAPS nasal pressure transducer and a ThermiSense oral/nasal thermistor (Salter Labs) and were worn as tolerated. Video recordings of the patient and respiratory plethysmography were obtained in synchrony with iEEG using the Nihon Kohden system. During the stimulation session, subjects wore the chest/abdominal plethysmography belt, nasal pressure transducer, and oral/nasal thermistor. Capillary oxyhemoglobin saturation $\left(\mathrm{SpO}_{2}\right)$ was recorded using a BluPro $\mathrm{SpO}_{2}$ sensor. A breath was defined as a complete inspiratory and expiratory cycle. Apnea durations were tabulated in Microsoft Excel based on measurements derived from NeuroWorkbench 
(Nihon Kohden). Central apnea was defined as at least 1 missed breath with a flattened airflow trace (oral/nasal thermistor or nasal pressure transducer) and verified by absence of chest wall movement measured with plethysmography belts or video. Trials were categorized as "apnea" when breathing was interrupted for the entire stimulation duration and as "transient apnea" when normal breathing resumed prior to the end of stimulation.

Experimental design and statistical analysis. Multiple stimulation trials were conducted at different forebrain electrode contact pairs (sites). The outcome of each stimulation trial was categorized as apnea, transient apnea, or no apnea and each stimulated site was categorized as apnea, transient apnea, or no apnea based on respiratory outcome elicited by the majority of trials. If stimulation of a site resulted in an equal distribution of respiratory outcomes over all trials at that site, it was categorized as the greater inhibitory effect of the 2 (e.g., if stimulation resulted in apnea and transient apnea in an equal number of trials, the site was categorized as apnea). If all 3 outcomes were observed with equal proportion, that site was categorized as the middle outcome (i.e., transient apnea); this occurred at only 1 site. After stimulation trials, all subjects except P447 (age 3) were asked to report any noticeable change in breathing, difficulty breathing, or changes in emotional state.

Statistics. Statistical and data analyses were conducted using Matlab (Mathworks), Prism 7.0 (Graphpad Software Inc.), and Microsoft Excel. For classification analyses, we used a multiclass support vector machine (66) with a 4-mm Gaussian kernel and 5-fold cross validation implemented in the Classification Learner App in Matlab R2018b (fitcecoc.m). The kernel standard width was selected to be $4 \mathrm{~mm}$ based on cross-validation performance.

Study approval. Experimental protocols were approved by the University of Iowa Institutional Review Board. Informed consent was obtained from the parents or legal guardians of all patients. Verbal assent was obtained from children 5 to 9 years of age, and written assent was obtained from all older children. No assent was obtained from the 3 year old, but she was tested in the company of her parents who had the option of terminating the experimental protocol at any time. Consent could be rescinded at any time without interfering with the child's clinical evaluation. Similarly, children could rescind assent at any time.

\section{Author contributions}

BJD, GISH, JAW, and AER conceived and designed the study. AER, CKK, GISH, AWS, DT, MAC, MAH, GBR, MS, JAW, and BJD acquired and/or analyzed data. BJD, AER, JAW, and MS drafted a significant portion of the manuscript or figures.

\section{Acknowledgments}

Support for this work was provided by the Roy J. Carver Charitable Trust (to BJD), the National Institute of Neurological Disorders and Stroke - Congress of Neurological Surgeons K12 Getch Scholar Award (to BJD), the National Institute of General Medical Sciences training grant T32 GM067795 (to GISH), and the National Institute of General Medical Sciences training grant T32 GM108540 (to AWS). The graphical abstract was created with BioRender.

Address correspondence to: Brian J. Dlouhy, Department of Neurosurgery, University of Iowa Carver College of Medicine, University of Iowa Stead Family Children's Hospital, 200 Hawkins Drive, Iowa City, Iowa 52242, USA. Phone: 319.356.2542; Email: brian-dlouhy@uiowa.edu.

1. Shorvon S, Tomson T. Sudden unexpected death in epilepsy. Lancet. 2011;378(9808):2028-2038.

2. Thurman DJ, Hesdorffer DC, French JA. Sudden unexpected death in epilepsy: assessing the public health burden. Epilepsia 2014;55(10):1479-1485.

3. Sveinsson O, Andersson T, Carlsson S, Tomson T. The incidence of SUDEP: A nationwide population-based cohort study. Neurology. 2017;89(2):170-177.

4. Sillanpää M, Shinnar S. Long-term mortality in childhood-onset epilepsy. N Engl J Med. 2010;363(26):2522-2529.

5. Dlouhy BJ, Gehlbach BK, Richerson GB. Sudden unexpected death in epilepsy: basic mechanisms and clinical implications for prevention. J Neurol Neurosurg Psychiatry. 2016;87(4):402-413.

6. Doumlele K, Friedman D, Buchhalter J, Donner EJ, Louik J, Devinsky O. Sudden unexpected death in epilepsy among patients with benign childhood epilepsy with centrotemporal spikes. JAMA Neurol. 2017;74(6):645-649.

7. Harden C, et al. Practice guideline summary: Sudden unexpected death in epilepsy incidence rates and risk factors: Report of the Guideline Development, Dissemination, and Implementation Subcommittee of the American Academy of Neurology and the American Epilepsy Society. Neurology. 2017;88(17):1674-1680. 
8. Verducci C, et al. SUDEP in the North American SUDEP Registry: The full spectrum of epilepsies. Neurology. 2019;93(3):e227-e236.

9. Ryvlin P, et al. Incidence and mechanisms of cardiorespiratory arrests in epilepsy monitoring units (MORTEMUS): a retrospective study. Lancet Neurol. 2013;12(10):966-977.

10. Kim Y, et al. Severe peri-ictal respiratory dysfunction is common in Dravet syndrome. J Clin Invest. 2018;128(3):1141-1153.

11. Bateman LM, Spitz M, Seyal M. Ictal hypoventilation contributes to cardiac arrhythmia and SUDEP: report on two deaths in video-EEG-monitored patients. Epilepsia. 2010;51(5):916-920.

12. Bateman LM, Li CS, Seyal M. Ictal hypoxemia in localization-related epilepsy: analysis of incidence, severity and risk factors. Brain. 2008;131(Pt 12):3239-3245.

13. Watanabe K, et al. Seizures with apnea in children. Pediatrics. 1982;70(1):87-90.

14. Nashef L, Walker F, Allen P, Sander JW, Shorvon SD, Fish DR. Apnoea and bradycardia during epileptic seizures: relation to sudden death in epilepsy. J Neurol Neurosurg Psychiatry. 1996;60(3):297-300.

15. Kaada BR, Jasper H. Respiratory responses to stimulation of temporal pole, insula, and hippocampal and limbic gyri in man. AMA Arch Neurol Psychiatry. 1952;68(5):609-619.

16. Dlouhy BJ, et al. Breathing inhibited when seizures spread to the amygdala and upon amygdala stimulation. J Neurosci. 2015;35(28):10281-10289.

17. Lacuey N, Hampson JP, Harper RM, Miller JP, Lhatoo S. Limbic and paralimbic structures driving ictal central apnea. Neurology. 2019;92(7):e655-e669.

18. McKay LC, Evans KC, Frackowiak RS, Corfield DR. Neural correlates of voluntary breathing in humans. J Appl Physiol. 2003;95(3):1170-1178.

19. Murphy K, et al. Cerebral areas associated with motor control of speech in humans. J Appl Physiol. 1997;83(5):1438-1447.

20. Masaoka Y, Homma I. Amygdala and emotional breathing in humans. Adv Exp Med Biol. 2004;551:9-14.

21. Rochet-Capellan A, Fuchs S. Changes in breathing while listening to read speech: the effect of reader and speech mode. Front Psychol. 2013;4:906.

22. Shea SA. Behavioural and arousal-related influences on breathing in humans. Exp Physiol. 1996;81(1):1-26.

23. Nobis WP, et al. The effect of seizure spread to the amygdala on respiration and onset of ictal central apnea. J Neurosurg. 2019:1-11.

24. Scherf KS, Smyth JM, Delgado MR. The amygdala: an agent of change in adolescent neural networks. Horm Behav. 2013;64(2):298-313.

25. Jiang Y, Tian Y, Wang Z. Age-related structural alterations in human amygdala networks: reflections on correlations between white matter structure and effective connectivity. Front Hum Neurosci. 2019;13:214.

26. Saygin ZM, et al. Structural connectivity of the developing human amygdala. PLoS One. 2015;10(4):e0125170.

27. Janak PH, Tye KM. From circuits to behaviour in the amygdala. Nature. 2015;517(7534):284-292.

28. Tyszka JM, Pauli WM. In vivo delineation of subdivisions of the human amygdaloid complex in a high-resolution group template. Hum Brain Mapp. 2016;37(11):3979-3998.

29. Nobis WP, et al. Amygdala-stimulation-induced apnea is attention and nasal-breathing dependent. Ann Neurol. 2018;83(3):460-471.

30. Gigliotti F. Mechanisms of dyspnea in healthy subjects. Multidiscip Respir Med. 2010;5(3):195-201.

31. Buchanan GF. Impaired $\mathrm{CO}_{2}$-induced arousal in SIDS and SUDEP. Trends Neurosci. 2019;42(4):242-250.

32. Richerson GB, Buchanan GF. The serotonin axis: shared mechanisms in seizures, depression, and SUDEP. Epilepsia. 2011;52 Suppl 1:28-38.

33. Hefti MM, et al. Hippocampal malformation associated with sudden death in early childhood: a neuropathologic study: Part 2 of the investigations of The San Diego SUDC Research Project. Forensic Sci Med Pathol. 2016;12(1):14-25.

34. Reis DJ, McHugh PR. Hypoxia as a cause of bradycardia during amygdala stimulation in monkey. Am J Physiol. 1968;214(3):601-610.

35. Feinstein JS, et al. Fear and panic in humans with bilateral amygdala damage. Nat Neurosci. 2013;16(3):270-272.

36. Maccotta L, et al. Impaired and facilitated functional networks in temporal lobe epilepsy. Neuroimage Clin. 2013;2:862-872.

37. Taugher RJ, et al. The amygdala differentially regulates defensive behaviors evoked by $\mathrm{CO}_{2}$. Behav Brain Res. 2020;377:112236.

38. Hagenaars MA, Oitzl M, Roelofs K. Updating freeze: aligning animal and human research. Neurosci Biobehav Rev. 2014;47:165-176.

39. Lacuey N, Zonjy B, Londono L, Lhatoo SD. Amygdala and hippocampus are symptomatogenic zones for central apneic seizures. Neurology. 2017;88(7):701-705.

40. Millhouse OE. The intercalated cells of the amygdala. J Comp Neurol. 1986;247(2):246-271.

41. Zikopoulos B, John YJ, García-Cabezas MÁ, Bunce JG, Barbas H. The intercalated nuclear complex of the primate amygdala. Neuroscience. 2016;330:267-290.

42. Nitecka L, Ben-Ari Y. Distribution of GABA-like immunoreactivity in the rat amygdaloid complex. J Comp Neurol. 1987;266(1):45-55.

43. Geerling JC, Loewy AD. Aldosterone-sensitive neurons in the nucleus of the solitary tract: bidirectional connections with the central nucleus of the amygdala. J Comp Neurol. 2006;497(4):646-657.

44. Hopkins DA, Holstege G. Amygdaloid projections to the mesencephalon, pons and medulla oblongata in the cat. Exp Brain Res. 1978;32(4):529-547

45. Moga MM, Gray TS. Peptidergic efferents from the intercalated nuclei of the amygdala to the parabrachial nucleus in the rat. Neurosci Lett. 1985;61(1-2):13-18.

46. Verner TA, Pilowsky PM, Goodchild AK. Retrograde projections to a discrete apneic site in the midline medulla oblongata of the rat. Brain Res. 2008;1208:128-136.

47. Jongen-Rêlo AL, Amaral DG. Evidence for a GABAergic projection from the central nucleus of the amygdala to the brainstem of the macaque monkey: a combined retrograde tracing and in situ hybridization study. Eur J Neurosci. 1998;10(9):2924-2933.

48. Yang CFK, Kim EJ, Callaway EM, Feldman JL. Monosynaptic projections to excitatory and inhibitory preBötzinger complex neurons. bioRxiv. https://doi.org/10.1101/694711. Published July 7, 2019. Accessed March 9, 2020

49. Petrovich GD, Risold PY, Swanson LW. Organization of projections from the basomedial nucleus of the amygdala: a PHAL study in the rat. J Comp Neurol. 1996;374(3):387-420.

50. Subramanian HH, Balnave RJ, Holstege G. The midbrain periaqueductal gray control of respiration. J Neurosci. 
2008;28(47):12274-12283.

51. Smith JC, Abdala AP, Borgmann A, Rybak IA, Paton JF. Brainstem respiratory networks: building blocks and microcircuits Trends Neurosci. 2013;36(3):152-162.

52. Sherman D, Worrell JW, Cui Y, Feldman JL. Optogenetic perturbation of preBötzinger complex inhibitory neurons modulates respiratory pattern. Nat Neurosci. 2015;18(3):408-414.

53. Smith JC, Ellenberger HH, Ballanyi K, Richter DW, Feldman JL. Pre-Bötzinger complex: a brainstem region that may generate respiratory rhythm in mammals. Science. 1991;254(5032):726-729.

54. McDonald AJ. Cytoarchitecture of the central amygdaloid nucleus of the rat. J Comp Neurol. 1982;208(4):401-418.

55. Pardo-Bellver C, Cádiz-Moretti B, Novejarque A, Martínez-García F, Lanuza E. Differential efferent projections of the anterior, posteroventral, and posterodorsal subdivisions of the medial amygdala in mice. Front Neuroanat. 2012;6:33.

56. Canteras NS, Simerly RB, Swanson LW. Organization of projections from the medial nucleus of the amygdala: a PHAL study in the rat. J Comp Neurol. 1995;360(2):213-245.

57. Buchanan GF, Richerson GB. Central serotonin neurons are required for arousal to CO2. Proc Natl Acad Sci USA. 2010;107(37):16354-16359.

58. Kaur S, et al. Glutamatergic signaling from the parabrachial nucleus plays a critical role in hypercapnic arousal. $J$ Neurosci. 2013;33(18):7627-7640.

59. Liotti M, et al. Brain responses associated with consciousness of breathlessness (air hunger). Proc Natl Acad Sci USA 2001;98(4):2035-2040.

60. Nathan SS, Sinha SR, Gordon B, Lesser RP, Thakor NV. Determination of current density distributions generated by electrical stimulation of the human cerebral cortex. Electroencephalogr Clin Neurophysiol. 1993;86(3):183-192.

61. Kawasaki H, et al. Processing of facial emotion in the human fusiform gyrus. J Cogn Neurosci. 2012;24(6):1358-1370.

62. Jenkinson M, Smith S. A global optimisation method for robust affine registration of brain images. Med Image Anal. 2001;5(2):143-156.

63. Oya H, Kawasaki H, Dahdaleh NS, Wemmie JA, Howard MA. Stereotactic atlas-based depth electrode localization in the human amygdala. Stereotact Funct Neurosurg. 2009;87(4):219-228.

64. Dale AM, Fischl B, Sereno MI. Cortical surface-based analysis. I. Segmentation and surface reconstruction. Neuroimage 1999;9(2):179-194.

65. Fish DR, Gloor P, Quesney FL, Olivier A. Clinical responses to electrical brain stimulation of the temporal and frontal lobes in patients with epilepsy. Pathophysiological implications. Brain. 1993;116(Pt 2):397-414.

66. Allwein EL, Schapire RE, Singer Y. Reducing multiclass to binary: A unifying approach for margin classifiers. J Mach Learn Res. 2000;1:113-141. 